\title{
Using 1D and 3D Anthropometric Data to Develop a Biofidelic Breast Cancer Patient Simulator
}

\author{
Daisy VEITCH ${ }^{a}$, Rachel DAWSON ${ }^{b}$, Harry OWEN ${ }^{b}$, Chris LEIGH ${ }^{a}$ \\ ${ }^{a}$ SHARP Dummies Pty Ltd, Belair (SA), Australia; \\ ${ }^{\mathrm{b}}$ Flinders Medical Centre, Bedford Park (SA), Australia
}

\begin{abstract}
Background

Early detection significantly reduces breast cancer mortality. Yet many medical students and doctors report they could improve their skills in clinical breast examination (CBE). Training programs using silicone breast simulators improve the lump detection rate [1]. Despite this, medical students and trainees typically perform low in breast examination scores [1]. This indicates current simulation models provide insufficient CBE training. In this study we have improved breast examination simulators by applying anthropometric data and selecting one very commonly occurring shape in the female population as a model.
\end{abstract}

Aims

To provide a breast model representative of the large size female population and more varied scenarios for breast lump palpation.

\section{Methods}

Comparing the 2002 National Size and Shape Survey of 1250 adult Australian women, ABS data and the Civilian American and European Surface Anthropometry Resource Project (CAESAR®), we selected individuals anthropometrically representative of the surveyed population. Combining one woman's body scan, computer-aided design, rapid prototype techniques and the latest biofidelic (lifelike) silicone technology we created an anatomically correct representation of a real world patient. This model requires trainees to learn that breast examination can be complex and require a multifaceted approach.

Results

A prototype representing women with larger breast size and higher body mass index (BMI) was developed. The individual selected is a large size woman of approximate BMI $30,82 \mathrm{~kg}$ and large cup size (D); by our analysis more than $50 \%$ of women are C cup or above.

\section{Conclusions}

Confident and competent breast palpation requires a life-size model that looks and feels lifelike. Currently available breast examination simulators don't model the size and shape of patients encountered. This impedes developing confidence and competence in health-care workers who need these skills. Lifelike look and feel require an anatomically correct, multi-layered soft breast construction incorporating palpable anatomical underlying features including tumors.

\section{Background}

Early detection of breast cancer significantly improves outcomes for patients by both increasing survival rates and reducing the invasiveness of treatment required [2]. Despite high quality mammography that can detect some cancers before they have become palpable the clinical breast examination (CBE) is still a core component of breast cancer detection and management [3]. The way CBE is taught and performed in Australia is not standardized [4] even though standardization has been reported to improve sensitivity and accuracy of lump detection [1]. This is why a standard breast patient simulator model needs to be used. In an extensive review of the performance and reporting of CBE undertaken for the American Cancer Society, McDonald et al. (2004) concluded "... it is reasonable to suggest that increased proficiency in CBE that leads to detection of smaller tumors may contribute to enhanced survival from breast cancer."[2].

A comprehensive CBE must include palpation, i.e., feeling the breasts correctly and with as little tissue as possible between the finger pads and the patient's rib cage. But there is no standardized procedure and it is thought this impacts adversely on cancer detection [2]. Competence (lump detection and identification) and confidence in CBE skills are improved by structured teaching that involves the use of silicone breast models and teaching associates [5]. 
Many medical students and doctors report they could improve their skills in clinical breast examination (CBE). Why? Most breast simulators are small and although patients in general and breasts in particular are getting larger (see below) no simulators are large. The way a CBE is conducted on a large breasted or obese patient is different to that on someone small breasted or not overweight.[6] Special patient positioning is used to minimise tissue thickness when palpating a large breast $[7,1]$, and also the breast area is larger, so the examination takes longer.[6] A recent commentary on simulation in medical education summarised the research thus: "...the recall of information and its application are best when it is taught and rehearsed in contexts similar to real life..."[8]. Consequently we studied the anthropometric data on Australian and North American populations to determine what large size patient simulators should be developed for optimal education and assessment.

Training programs using silicone breast simulators improve the lump detection rate [1]. Despite this, medical students and trainees typically perform low in breast examination scores [1]. This indicates current simulation models provide insufficient CBE training. There will be many reasons for this but lack of practice on an appropriate simulator will be an important one. Learning how to palpate the breast requires a life-size model that looks and feels authentic. For example, it has been reported that students do not use enough pressure during palpation. Varying amounts of pressure should be used to identify and locate lesions in the breast. This means that the materials used in a simulator need to deform in a similar way to real breast tissue and should be on a chest wall so that the trainee can feel ribs and know they are using appropriate pressure to identify the deepest lesions. Breast palpation includes the axilla and above and below the clavicle, so the model requires these structures for the whole sequence to be learnt.

It is important that medical students learn CBE well during medical school. In a study of medical practitioners it was revealed that individuals who felt they had been taught digital rectal examination well in medical school were more likely to perform this examination on their patients [9]. The knowledge gained from this study may be generalised to the practice of CBE as yet another type of intimate examination. Current evidence reveals that health care workers do not feel confident in CBE and would welcome further training [2]. Studies of medical students have revealed similar trends, with students reporting they feel underprepared and require further training [10]. In this study we have developed a breast examination simulator by applying anthropometric data and selecting the most commonly occurring shape in the large size female population as a base to build the model.

\section{Method}

The need for a biofidelic model of large size women does not answer the question of how such a model is to be designed. The purpose of this section is to describe the process by which such a design was achieved. This requires the combination of anthropometric data of a number of kinds from a variety of sources. The statistical analysis of these data informs the design process.

\subsection{Anthropometric trends and analysis}

In the last 30 years obesity has become a major public health issue [11] and more than half the adult population in Australia and many other countries are now overweight or obese. The effect of increasing weight on diabetes and cardiovascular disease is widely appreciated but the increased prevalence of obesity also impacts on breast cancer risk and detection. Higher Body Mass Index (BMI) is correlated with an increased risk of breast cancer, and women whose BMI is $\geq 30$ kilograms $/ \mathrm{m} 2$ face a threefold increase in breast cancer risk [12]. Also an inverse relationship between CBE sensitivity and increased body weight has been reported [13]. Health professionals need specific help to develop their palpation skills to detect lumps in obese patients [6], and with obesity in children now common [14] this will be a continuing training need. Another consequence of overweight and obesity is that these women are less likely to present to their doctor for intimate examinations including CBE [15], so health professionals will need to be proactive and offer CBE for screening when these patients present for other reasons such as obtaining contraception. Poor confidence in CBE skills is a barrier to this [16] and is compounded when the patient is obese [17].

There are several reports of health professionals perceiving the need for more training in CBE generally [18] and of perceived inadequacies in performing CBE on obese patients [17]. Good initial training must include adapting the examination to patients with large breasts, obesity or morbid obesity and this needs to be followed up by programs focussing on skill maintenance [19].

Precise measurements of breast size can be derived from three-dimensional (3D) body scans and manually collected one-dimensional (1D) data. There is no Australian database for the 3D measurements, but there are height and weight mean averages: Australian data for women has the population mean weight at $67.7 \mathrm{~kg}$ [20] and mean height at $1639 \mathrm{~mm}$ [21]. This population data was matched to an analysis of a raw data subset from the Civilian American and European Surface 
Anthropometry Resource (CAESAR®)[22] $(n=939)$. The subset analysed were female, aged 18-65, civilian, white women living in the US, mean weight $68.7 \mathrm{~kg}$ and mean height $1651 \mathrm{~mm}$, which is comparable to the Australian means. The US subset matched to Australian data revealed that $52 \%$ have a breast cup size classification of $C$ or above (see the pale squares in Figure 1). The bra sizing classification chosen for this analysis is the "International" sizing in which the women categorized as C cup and above are those with a difference of 6 inches and greater in their bust and under bust measurement [23]. Although millimetres are the international standard for measurement, the International bra sizing is quoted in inches, so inches are used for ease of analysis here.

It should be noted that 174 women (nearly $20 \%$ ) were too large for any standard size bra in this prediction chart, having a bust circumference $>42$ inches or an under bust of $>36.5$ inches and almost all of these have breast size of $\mathrm{C}$ cup or greater.

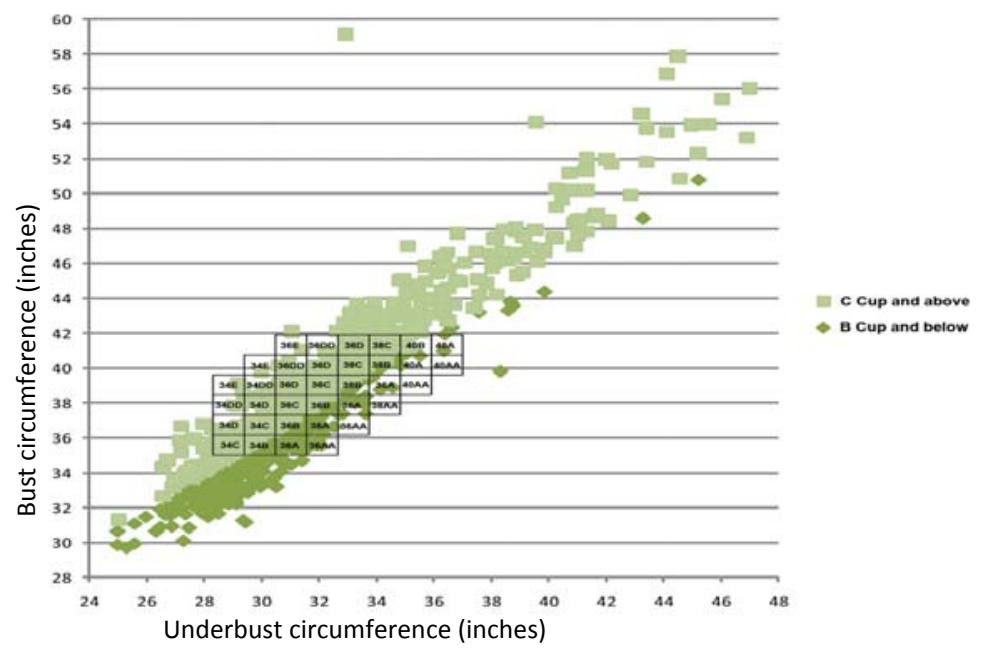

Fig. 1. Distribution of cup sizes with overlay of International bra sizing prediction chart

According to a recent report from a major lingerie manufacturer, bra cup size in Australia has tripled over the last 50 years [24]. The average cup size was 10B in 1960, 12B in 2000 and 14C in 2010, which might mean women who are not overweight may be developing larger breasts, or it might indicate an allometric phenomenon. Huxley and Teissier define allometry as, "the growth of a part of a body at a different rate from that of a body as a whole..." Allometry for bust and underbust circumference (see Figure 1) shows that women of larger circumferences usually have a larger cup size. Thus increased obesity in the population results in an increased proportion of women with large cup size (see Figure 2). We draw this conclusion from the assumption that increased circumferences are linked to increased weight. The pale squares in Figure 1 indicate individuals who have a C-cup or above and the dark diamonds represent B-cup or below. The division line is skewed. This shows that breast cup size $C$ and above is much more frequent in large circumference women. Therefore as the population circumference mean increases more bras of cup size $C$ and above will be sold.

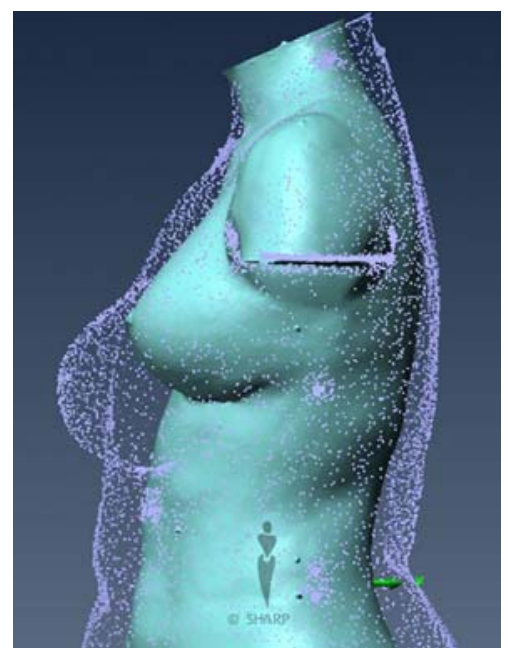

Fig. 2. The allometric phenomenon - data points from a body scan of an obese patient superimposed on the scan of a woman of ideal bodyweight to show how obesity and cup size changes the relative dimensions of the body. 


\subsection{Approach}

The statistical method for this project was developed by the USAF CARD Lab (United States Air Force Computerized Anthropometric Research and Design Laboratory) with SHARP (Surface Human Anatomy Replication of People) Dummies Pty Ltd. SHARP Dummies is a private research company that specializes in anthropometric research applied to design such as manikins. This method produced SHARP's first biofidelic manikin for the apparel industry. The current project to develop the breast cancer large manikin shape was based on a second apparel collaboration with US based companies. It uses body scan data from a representative sub-population of large size women to create more realistic models. Using CAESAR® data we applied the following measuring and manufacturing materials and methodology.

\subsection{Body selection}

Using 1D data such as height and weight is not sufficient to define body shape. This is because same weight and height women can vary significantly in the distribution of adipose tissue and thus circumferences (see Figure 3). Therefore more detailed measurements such as bust, underbust, waist and hip circumference are required to define shape.

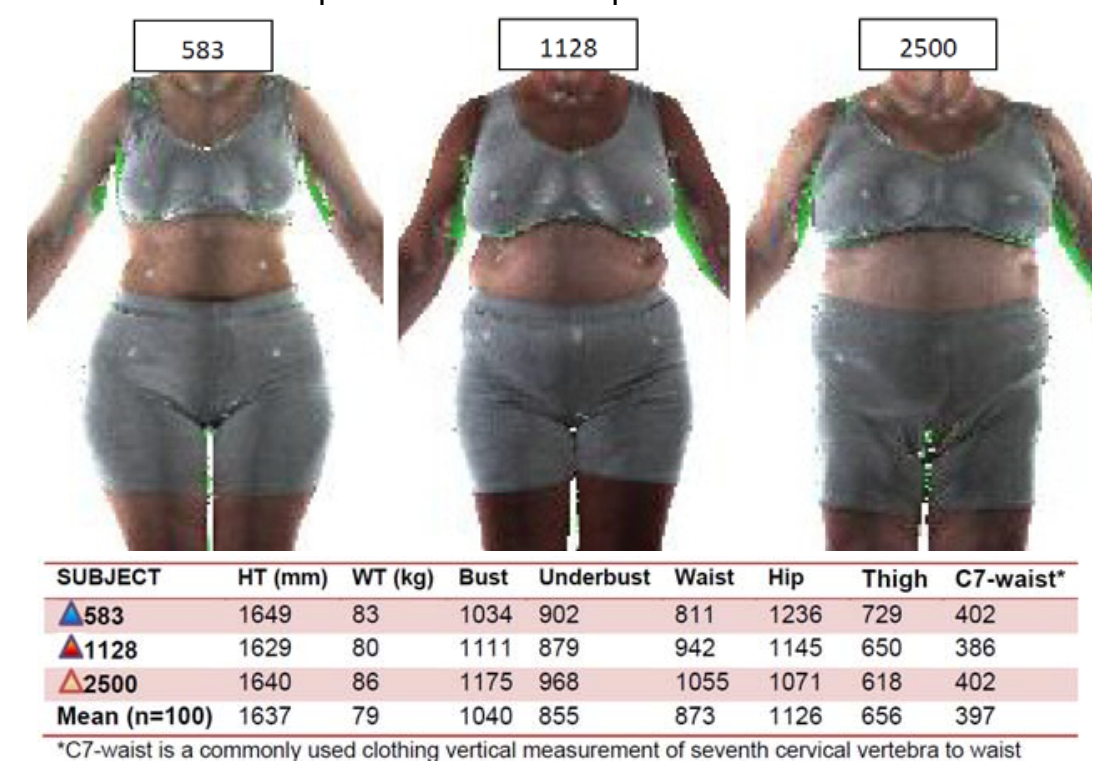

Fig.3. Scanned data from three figures of similar height and weight but with different circumferences, hence body shape are shown with measurements and means of the subset $(n=100)$. Circumferences are in millimetres and weight in kilograms. Locations are linked to Figure 4 by the different coloured triangles.

Bivariate plots have been used to select the most commonly occurring body shapes (see Figure 4). Each blue dot in the plot represents an individual's measurements. We selected an individual of weight $82 \mathrm{~kg}$ and height $1639 \mathrm{~mm}$ who was in the centre of the waist vs. hip scatter plot (and also underbust vs. bust). These 1D measurements have been used in conjunction with other factors, such as symmetry, assessed visually using the images of 3D scans.

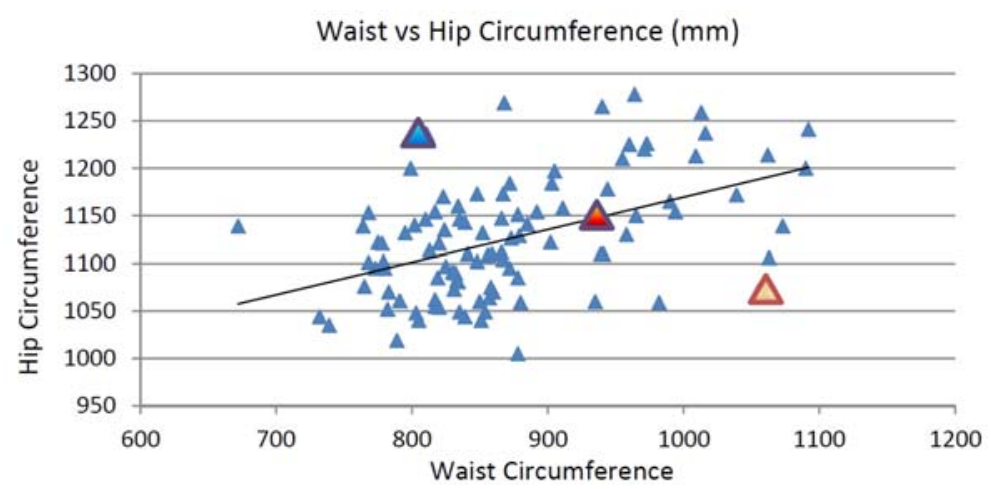

Fig. 4. Bivariate plot of waist vs. hip circumference of 100 North American civilian women who weigh between $70-95 \mathrm{~kg}$ and whose height is from 1620 to $1650 \mathrm{~mm}$. 
Analysis of individual data shows subjects are within $3 \mathrm{~kg}$ of each other and $20 \mathrm{~mm}$ in height. However the waist minus hip shows subject 583 is "curvy" subject, 1128's shape is "most common" (on the line of best fit) and subject 2500 is "straight" [25]. For this study we selected an individual from the centre of the distribution.

\section{Results}

SHARP Dummies and University of Adelaide undertook a National Size and Shape Survey of 1250 adult women in 2002 [26]. The population mean for height and weight results of this survey, along with those of the Australian Bureau of Statistics [20,21], were matched with CAESAR ${ }^{\circledR}$ height and weight data. This comparison of Australian vs. North American data shows that these populations are very similar, so we made the decision to apply North American data to the Australian population. The high quality, usability and access to $3 \mathrm{D}$ scans provided by the North American CAESAR ${ }^{\circ}$ data enabled the detailed analysis in this project. Only a subset of these data was used: large size females of weight range $70-95 \mathrm{~kg}$ of average height $1620-1650 \mathrm{~mm}$ tall. There were 100 women in this category. This subset enabled us to create a manikin representative of a large size real world patient. Once we selected a representative individual we cleaned their 3D data using computer-aided design (CAD) and checked the measurements (see Figure 5). The selected shape was made 'watertight' for rapid prototyping because any 'holes' or gaps in the 3D data cause milling errors. We then made fiberglass moulds or negatives of the master shape or positive so multiple copies of the manikin can be produced.

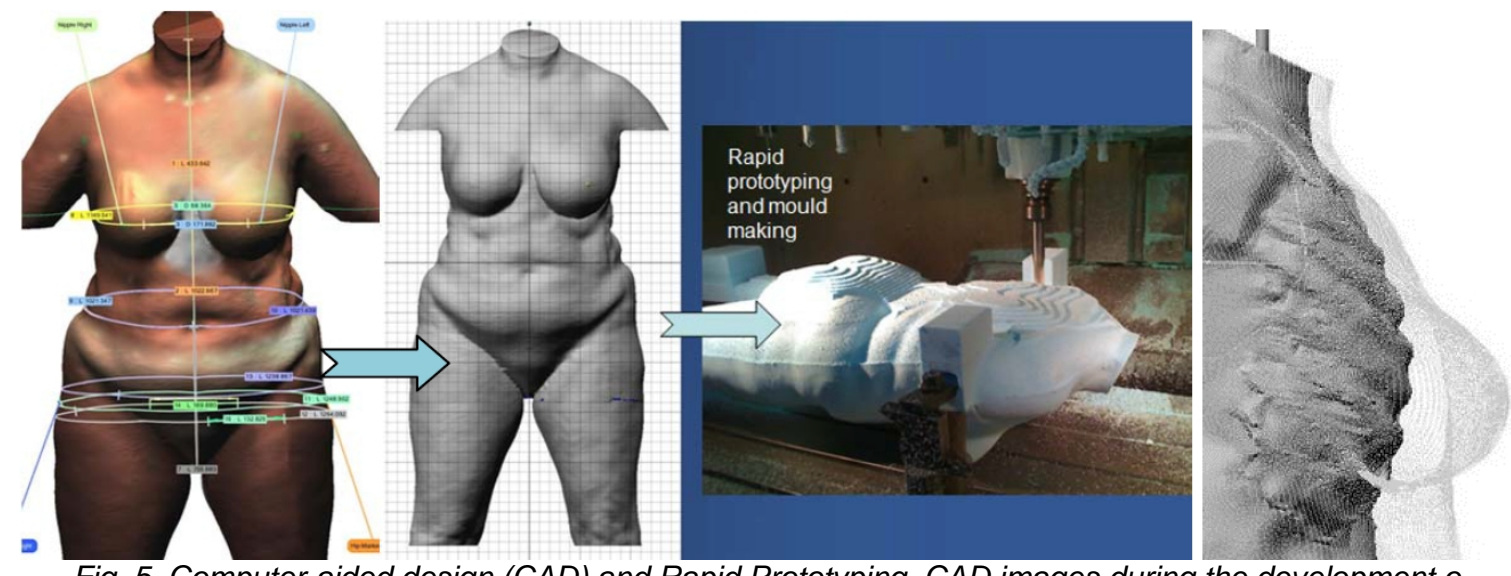

Fig. 5. Computer-aided design (CAD) and Rapid Prototyping. CAD images during the development o the lifelike breast model and side view of the rib cage overlaid with adipose tissue shape.

We added underlying anatomical features, such as the skeleton, with CAD (see Figure 5). These features were manufactured separately and assembled later. Extensive testing of materials for feel, dimensional stability and durability were conducted for skin, adipose tissue, rib, cartilage, lungs and tumors. Materials were then selected that mimic the lifelike feel of the torso (skeleton and muscles) and breasts (normal breast tissue, tumours and lymph nodes). The manikin was constructed from multiple layers of the materials to resemble a real body to give optimal results in look, colour, texture and feel (see Figure 6). Six clinicians (including two practising breast surgeons) reviewed the model and reported the breast and tumours felt lifelike. The breast changes throughout life and two commented that the simulator had characteristics of a postmenopausal breast.

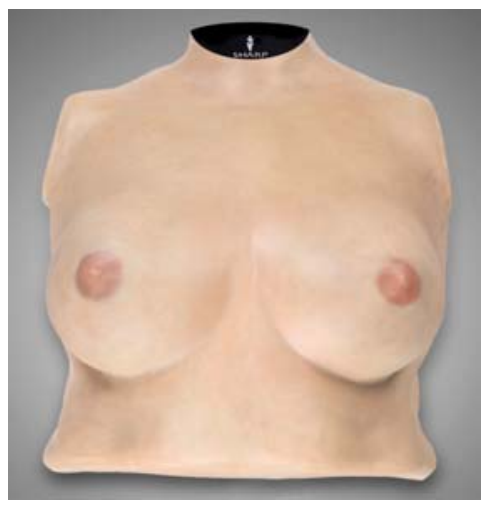

Fig. 6. Completed prototype manikin made from silicone skin, soft adipose tissue, rigid rib cage, nodular lumps and tumors. 
This multi-layering of the biofidelic model enables the trainee to feel the complexity of the underlying anatomical structure. This trains the fingers to detect both normal anatomy and any lesions, such as tumors, under a large breast, making the learning experience more representative of a complex real patient breast examination.

Fourteen tumors have been inserted in a variety of locations, including the axillae and high on the chest wall near the clavicle, which could occur in real life but be outside the range of a mammogram, making CBE an essential skill for early detection. Tumors also have been inserted deep in the breast where they cannot be detected using $\mathrm{CBE}$, giving the trainee, as one of the training outcomes, some degree of uncertainty as to whether a lesion is present or not. The correct action then would be referral of the patient for mammogram. This combination of tumors in the manikin is designed to create a sense in the trainee of the need for further detective work to achieve a reliable outcome. Training should be complex enough to reinforce the three pillars of the 'triple test': Mammography, Biopsy, and CBE, which, when fully executed, will detect a breast cancer with $99.6 \%$ sensitivity [3]. In addition it should highlight the need to take extra care and time in examining large breasted patients because of the higher degree of difficulty with these patients. Specialist training should be provided as there is some evidence physicians may be reluctant to perform CBE on large women, perceiving such examinations to be more difficult; large patient simulators provide the opportunity for this training [6].

\section{Discussion and conclusion}

Obese patients are more likely to develop breast cancer but are less likely to present with breast symptoms and, if they do, breast lumps are more difficult to detect because harder to feel. Some studies using silicone models suggest that, with training, lumps as small as $3 \mathrm{~mm}$ can be palpated, but not reliably, and at the cost of reduced specificity [27].

Studies of health professionals reveal many have little recent experience of CBE and lack of confidence impairs their performance [28]. In many areas of medicine, lack of confidence is most often ascribed to the small numbers of cases encountered during training.

The time taken to examine a large breast will be longer than a small breast. Silk and McTigue (2011) have suggested more than 3 minutes per breast is required, but following the now recommended technique using small circular movements of the first three fingers and a vertical strip pattern, it requires 3-4 minutes for an average breast [2]. This is much longer than may be happening in clinical practice.

Practising palpation on large breast simulators will help trainees become comfortable in taking what seems like a long time for a thorough examination. They should also rehearse the explanation they give about the procedure and be prepared for the extra time needed to reposition the obese patient to facilitate the examination [7].

Small size reduces the cost of making and transporting the models and space required for storage, but it also makes them less useful for training and assessment. Large breasted patient simulators are needed to provide a more realistic representation of the current population and so improve CBE teaching. A chest wall and ribs allow realistic feeling so that one can practice determining the pressure required for deep palpation, and clavicles and axillae are required for thorough palpation. Some health professionals worry about hurting the patient and so won't apply enough pressure to detect abnormalities in deeper tissues [5]. A realistic skin feel and texture allows for practising palpation technique with the pads of three fingers.

We were disturbed to discover that some students thought mammography was an alternative to CBE, and this is not an isolated finding. Apparently some GPs do not place value on the skill of CBE, and are willing to rely on mammograms for the detection of lesions [4]. Mammography misses $10 \%$ to $20 \%$ of clinically palpable breast cancers [29]. This highlights the need to emphasize the continuing importance of CBE in early breast cancer detection to medical students, and the need for more training opportunities for both students and graduates.

There are currently gaps in the availability of resources to teach the skills required for the investigation of a new breast symptom, and this is not helped by a lack of standardization in teaching practice. An increasingly obese population with increased risk of breast cancer intensifies the need for awareness of this issue and for the skills required to effectively investigate a new breast symptom, particularly in large breasts. Thorough training in CBE at medical school is necessary for all students, but more experienced health professionals may also need opportunities to practise CBE, particularly on a larger model.

Sensitivity of lesion detection can be improved using basic silicone models [30] but a realistic model of the upper half of the body at least is required for the systematic teaching of breast palpation technique. Acquiring competence requires authentic simulators providing a range of sizes that includes very large. 
Teaching inspection and palpation of the breast help trainees become comfortable with intimate examinations, but this rarely happens with large patients. Obese volunteers need to be deliberately included in medical training [6].

The development of the large size biofidelic simulator described here attempts to fill this training gap in the attention given to large size patients. It provides a representative model based on the population who need it; they have increased cancer rate and are becoming increasingly common in the population but they have been previously avoided. Reproducible multiple copies create the essential base for the standardized model upon which a standardized CBE training module can be developed.

\section{Acknowledgements}

Design and manufacture of the breast models were funded through the Flinders University Collaborative Research Grants Scheme. Dr S Kirubakaran, Dr A Vnuk and Dr M Bochner provided CBE training and advice on using breast models in clinical skills training.

A version of this paper has been published in Ergonomics Australia - HFESA 2011 Conference

Edition, 2011 11:45 <http://www.ergonomics.org.au/resource_library/journal.aspx>

\section{References}

1. Saslow D, Hannan J, Osuch J, Alciati M, Baines C, Barton M, Bobo J, Coleman C, Dolan M, Gaumer G, Kopans D, Kutner S, Lane D, Lawson H, Meissner H, Moorman C, Pennypacker H, Pierce P, Sciandra E, Smith R, Coates R. 2004. Clinical Breast Examination: Practical Recommendations for Optimizing Performance and Reporting. CA Cancer Journal for Clinicians 54:327-344.

2. McDonald S, Saslow D, Alciati M. 2004. Performance and Reporting of Clinical Breast Examination: A Review of the Literature. CA Cancer Journal for Clinicians 54(6):345-361.

3. Irwig L, Macaskill P, Houssami N. 2002. Evidence relevant to the investigation of breast symptoms: the triple test. The Breast 11:215-220.

4. Thistlewaite J. 2007. Clinical Breast Examination for asymptomatic women. Australian Family Physician. 36:145-149.

5. Steiner E, Austin DF, Prouser NC. 2008. Detection and description of small breast masses by residents trained using a standardized clinical breast exam curriculum. Journal of General Internal Medicine 23(2):129-134.

6. Silk AW, McTigue KM. 2011. Re-examining the physical examination for obese patients. Journal of the American Medical Association 305(2):193-194.

7. Barton MB, Harris R, Fletcher SW. 1999. Does this patient have breast cancer? Journal of the American Medical Association. 282(13): 1270-1280.

8. Kahn K, Pattison T, Sherwood S. 2011. Simulation in medical education. Medical Teacher 33:13.

9. Hennigan T, Franks P, Hocken D, Allen-Mersh T. 1990. Rectal examination in general practice. British Medical Journal 301: 478-480.

10. Kann P, Lane D. 1998. Breast cancer screening knowledge and skills of students upon entering and exiting a medical school. Academic Medicine 73(8): 904-906.

11. Access Economics. The Economic Costs of Obesity, Diabetes Australia, October, Canberra 2006.

12. Montazeri A, Sadighi J, Farzadi F, Maftoon F, Vahdaninia M, Ansari M, Sajadian A, Ebrahimi M, Haghighat S, Harirchi I. 2008. Weight, Height, body mass index and risk of breast cancer in postmenopausal women: a case-control study. BMC Cancer 8: 278-285.

13. Oestreicher N, White E, Lehman C, Mandelson M, Porter P, Taplin S. 2002. Predictors of sensitivity of clinical breast examination (CBE). Breast Cancer Research \& Treatment 76(1):7381.

14. Magarey AM, Daniels LA, Boulton JC. 2001. Prevalence of overweight and obesity in Australian children and adolescents: reassessment of 1985 and 1995 data against new standard international definitions. Medical Journal of Australia 174: 561-564.

15. Fontaine K, Bartlett S. 2002. Access and use of medical care among obese persons. Obesity Research 8:403-406.

16. Lanotti RJ, Finney LJ, Sander AA, De Leon JM. 2002. Effect of clinical breast examination training on practitioner's perceived competence. Cancer Detection and Prevention 26:146-148. 
17. Ferrante JM, Fyffe DC, Vega MS, Piasecki AK, Ohman-Strickland PA, Crabtree BF. 2010. Family Physicians' Barriers to Cancer Screening in Extremely Obese Patients. Obesity (Silver Spring) 18(6):1153-1159.

18. Pennypacker HS, Naylor L, Sander AA, Goldstein MK. 1999. Why can't we do better breast examinations? Nurse Practice Forum 10(3):122-128.

19. Chalabian J, Garman K, Wallace P, Dunnington G. 1996. Clinical breast evaluation skills of house officers and students. American Journal of Surgery 62(10):840-845.

20. 2004-5, Overweight and Obesity in Adults, Australian Bureau of Statistics, 25 Jan 2008, p 5.

21. 1995, How do Australians Measure Up, Australian Bureau of Statistics, 6 Oct 1998, p 11.

22. Data from World Engineering Anthropometry Resource (WEAR) (https://wear.istdayton.com)

23. Wright, M.C.M. Graphical analysis of bra size calculation procedures, International Journal of Clothing Science and Technology, Vol. 14 No. 1, 2002, pp. $41 \pm 45$.

24. Woods E. 2010. Boom and bust. Sydney Morning Herald on-line edition www.smh.com.au/lifestyle/lifematters/boom--bust-20100719-10gx9.html

25. Robinette K., 2005, American Standards and Testing Materials (ASTM) D-13 presentation, Washington DC, USA

26. Henneberg M., and Veitch D., "Australian Size Survey," ISAK Kinanthreport Vol. XVI, No 1, 2003, p34.

27. Fletcher SW, O'Malley MS, Pilgrim CA, Gonzalez JJ. 1989. How do women compare with internal medicine residents in lump detection? Journal of General Internal Medicine 4: 277-283.

28. Wiecha JM, Gann P. 1993. Provider confidence in breast examination. Family Practice Research Journal 13:37-41.

29. Barlow WE, Lehman CD, Zheng Y, Ballard-Barbash R, Yankaskas BC, Cutter GR, Carney PA, Geller BM, Rosenberg R, Kerlikowske K, Weaver DL, Taplin SH. 2002. Performance of diagnostic mammography for women with signs or symptoms of breast cancer. Journal of the National Cancer Institute 94(15):1151-1159.

30. McDermott MM, Dolan NC, Huang J, Reifler D, Rademaker AW. 1996. Lump detection is enhanced in silicone models simulating postmenopausal breast tissue. Journal of General Internal Medicine 11(2):112-114. 